\title{
RX J0513.9-6951 AND V SAGITTAE: ACCRETION WIND EVOLUTION, A KEY EVOLUTIONARY PROCESS TO TYPE IA SUPERNOVAE
}

\author{
Izumi Hachisu $^{1}$ \\ RESUMEN
}

Se propone un nucvo modelo auto-consistente de las variaciones de larga duración de la curva de luz de las fuentes supersuaves de rayos X RX J0513.9-6951 y V Sge, basado un un modelo de viento ópticamente espeso de las cnanas blancas acrecientes (WDs). Cuando la tasa de masa acrecida excede el crítico valor de $\sim 1 \times 10^{-6} M ., \mathrm{yr}^{-1}$, fuertes vientos ópticamente espesos comienzan a surgir desde la enana blanca, de tal manera que se evita la formación de una envolvente común. La WD puede acrecer y quemar material rico (n) hidrógeno en la superficie de la enana blanca en la tasa crítica. El exceso de materia transferida a la WD por encima de ess valor crítico es expelido por vientos. Esto se denomina "accretion wind evolution". Este proceso de eyección, sin embargo, ocurre intermitentemente debido a que la transferencia de masa es atenuada por fuertes vientos: estos fuertes vientos colisionan con la superficie de la secundaria desnudándola de su capa superficial. Formulando propiamente este efecto de pérdida de masa y el consiguiente decaimiento del ritmo de transferencia de masa, somos capaces de reproducir la transición entre los estados de alta luminosidad en el óptico/presencia de rayos X y baja luminosidad en el óptico/ausencia de rayos X, de las fuentes de rayos X supersuaves RX J0513.9-6951 y V Sge. Esos dos objetos son los primeros ejemplos de "acretion wind evolution", lo cual es una clave en el proceso evolutivo de la trayectoria de evolución recién desarrollada por las supernovas de tipo Ia.

\section{ABSTRACT}

A new self-sustained model for long-term light-curve variations of the supersoft X-ray sources RX J0513.9-6951 and V Sge is proposed based on an optically thick wind model of mass-accreting white dwarfs (WDs). When the nliass accretion rate to a WD exceeds the critical rate of $\sim 1 \times 10^{-6} M_{\odot} \mathrm{yr}^{-1}$, optically thick strong winds begin to blow from the WD so that a formation of common envelope is avoided. The WD can accrete and burn hydrogen-rich matter on the surface of the WD at the critical rate. The excess matter transferred to the WD above the critical rate is expelled by winds. This is called "accretion wind evolution." This ejection process, however, occurs intermittently becausc the mass transfer is attenuated by strong winds: the strong winds collide with the secondary surface and strip off the surface layer of the secondary. Properly formulating the massstripping cff c't and the ensuing decay of mass-transfer rate, we are able to reproduce the transition between the optical high/X-ray-off and optical low/X-ray-on states of the supersoft X-ray sources RX J0513.9-6951 and $V$ Sge. Thus these two objects are the first examples of accretion wind evolution, which is a key evolutionary process in a recently developed evolutionary path to Type Ia supernovae.

Key Words: BINARIES: CLOSE - STARS: INDIVIDUAL (RX J0513.9-6951, V SGE) - STARS: WINDS, OUTFLOWS - X-RAYS: STARS

\section{INTRODUCTION}

It has been frequently discussed that supersoft $\mathrm{X}$-ray sources are progenitors of Type Ia supernovae (SNe Ia). One promising evolutionary model of SNe Ia has been proposed by Hachisu, Kato, \& Nomoto (1999a) and Hachisu et al. (1999b). They found theoretically that mass-accreting white dwarfs (WDs) blow strong winds when the mass accretion to the WD exceeds a critical rate. i.e., $\dot{M}_{\text {icc }}>\dot{M}_{\text {cr }} \sim$

\footnotetext{
${ }^{1}$ Dept. of Earth Science and Astronomy, University of Tokyo, 15.3-8902, Japan. email: hachisu@chianti.c.utokyo.ac.jp
}

$1 \times 10^{-6} M_{\odot} \mathrm{yr}^{-1}$ (Hachisu, Kato, \& Nomoto 1996; Kato \& Hachisu 1994). The so-called common envelope evolution does not occur (see Hachisu \& Kato 2001, for a recent summary). The WD accretes hydrogen-rich matter and burns it on the surface of the WD at the critical rate of $\dot{M}_{\mathrm{cr}}$. The excess matter transferred above the critical rate is expelled by winds, i.e.. $\dot{M}_{\text {wind }}=\dot{M}_{\text {acc }}-\dot{M}_{\text {cr. }}$. In such a situation, the WD can grow to the Chandrasekhar mass and eventually explode as an SN Ia ( $\mathrm{Li} \&$ van den Heuvel 1997). This is called "accretion wind evolution" (Hachisu \& Kato 2001), as against the com- 
mon envelope evolution. Thus, accretion wind evolution is a key evolutionary process in the production of Type Ia supernovae.

Accretion wind evolution was predicted to exist in binary systems but no such systems have been identified yet. Here we have reached the conclusion that RX J0513.9-6951 and V Sge are the first examples of such accretion wind evolution.

\section{MODELING THE LMC SUPERSOFT SOURCE RX J0513.9-6951}

The Large Magellanic Cloud (LMC) transient supersoft X-ray source RX J0513.9-6951 (hereafter RX J0513) was discovered by ROSAT (Schaeidt, Hasinger, \& Truemper 1993) and has been extensively observed (e.g., Reinsch et al. 2000, for recent progress). Soon after the X-ray discovery, it was optically identified as a binary with an orbital period of 0.76 days (Pakull et al. 1993). Its remarkable observational features are summarized as follows: (1) Optical monitoring of RX J0513 shows quasi-regular transitions between high $(V \sim 16.6)$ and low $(V \sim 17.4)$ states (Alcock et al. 1996). (2) The duration of the optical high state is $\sim 60-150$ days while the low states last for $\sim 40$ days (see, e.g., Fig. 1 of Cowley et al. 2002, for recent observational summary). (3) Copious supersoft X-rays $(\sim 30-40$ $\mathrm{eV}$ ) were detected only in the optical low state (e.g., Schaeidt et al. 1993; Reinsch et al. 1996; Southwell et al. 1996). (4) The transitions between X-ray-off and X-ray-on are very rapid, about one day or so, while the optical transitions from high to low occur over several days (e.g., Reinsch et al. 2000). The transition mechanism between high and low states has not been fully elucidated yet, although a few ideas were proposed so far (see, e.g., Reinsch ct al. 2000, for a recent progress).

Long-term variations in the optical light curves of RX J0513 are considered as a limit cycle (e.g., Reinsch et al. 2000). We adopt a binary system consisting of a WD, a disk around the WD, and a lobe-filling main-sequence (MS) companion. A circular orbit is assumed. An essential thing is that WDs blow an optically thick, strong wind when the rate of mass accretion to the WD exceeds the critical rate, i.e., $\dot{M}_{\text {acc }}>\dot{M}_{\mathrm{cr}} \sim 1 \times 10^{-6} M_{\odot} \mathrm{yr}^{-1}$ (Hachisu et al. 1996; Hachisu \& Kato 2001) as illustrated in Figure 1. If the MS companion is $\sim 2-3 M_{6}$, the mass-transfer rate from the MS, $\dot{M}_{\mathrm{MS}}$, is as large as a few times $10^{-6} M_{C} \mathrm{yr}^{-1}$ or more (e.g., Li \& van den Heuvel 1997).

The time-evolution of our limit cycle model is schematicaily summarized as follows in Figure 2 (see

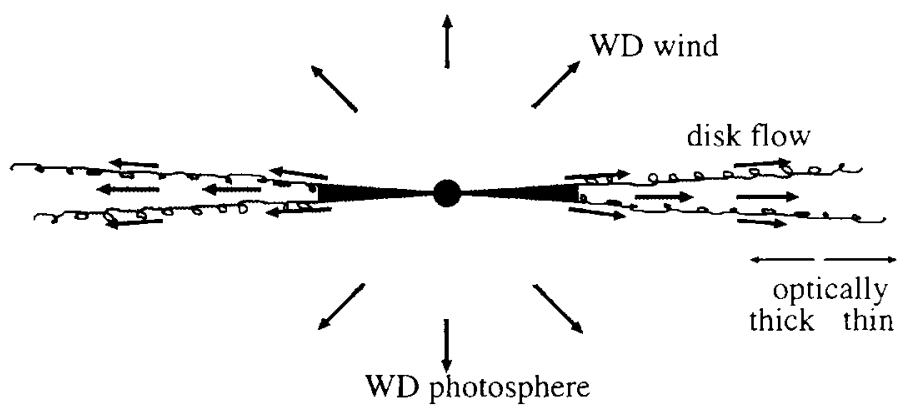

Fig. 1. Optically thick part of the disk expands during the wind phase because its surface layer is blown off.

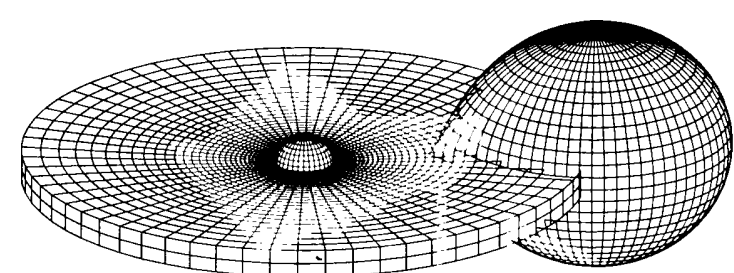

(a) optically thick winl in:?

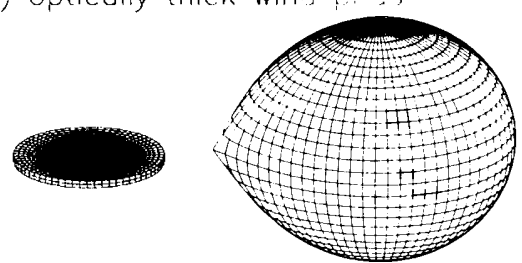

(b) just ofter the win: stops

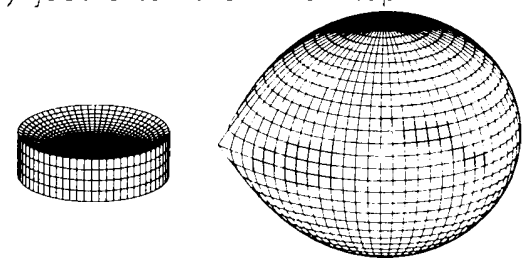

(c) mass acireiur mukes

Fig. 2. Optically thick surfaces of our bincry model for (a) the wind phase, (b) just after the wind stops. and (c) the massive mass transfer.

also Hachisu \& Kato 2003b). A rapid mass accretion to the WD begins. The mass of the WD envelope rapidly increases, and the WD envelope expands to blow an optically thick, strong wind. The strong wind causes a quick rise of $V$ magnitude. because the disk expands from a few to several times the previous size, that is, the disk expands from the configuration shown in Figure 22. to that shown in Figure 2a. Because the irradiated disk is the main source in the optical light, this expansion of the disk area causes a magnitude rise in the optical light. The wind mass loss rate increase's from zero to $M_{\text {wind }} \sim 1 \times 10^{--x} M . y^{-1}$ in a dar, which is milsisive' enough to alssorb supersoft $\mathrm{X}$-rays. Thus, this phase is the optical high and $\mathrm{X}$-ray-off state.

The strong wind hits the MIS companion and then strips off its surface laser. The stripped-off gals is 

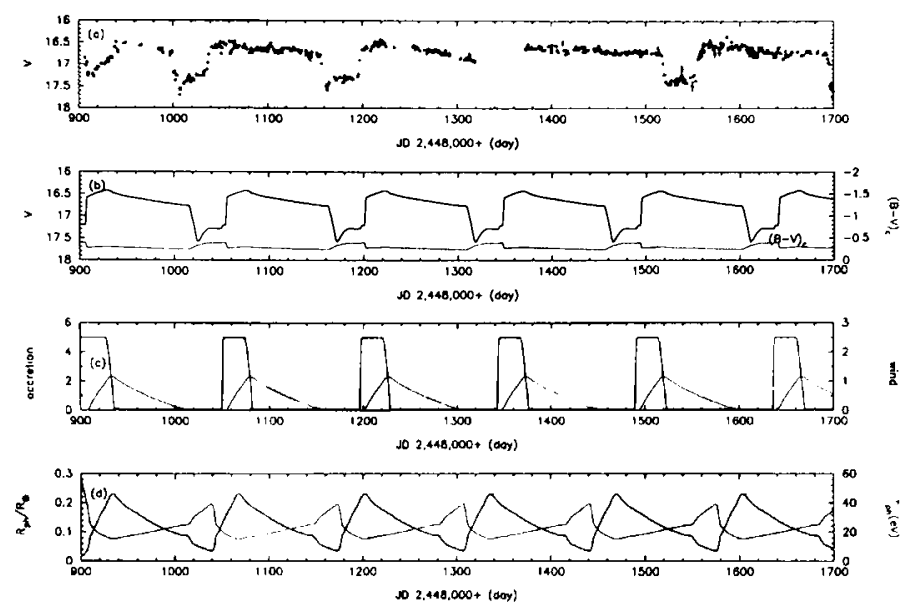

Fig. 3. Long term light curve of the LMC supersoft X-ray source RX J0513.9-6951 for (a) MACHO observation (taken from Alcock et al. 1996), (b) our modeled light curve, (c) mass accretion rate and wind mass loss rate in units of $10^{-6} M \ldots \mathrm{yr}^{-1}$, and (d) photospheric radius $\left(R_{.}\right)$ and temperature $(\mathrm{eV})$.
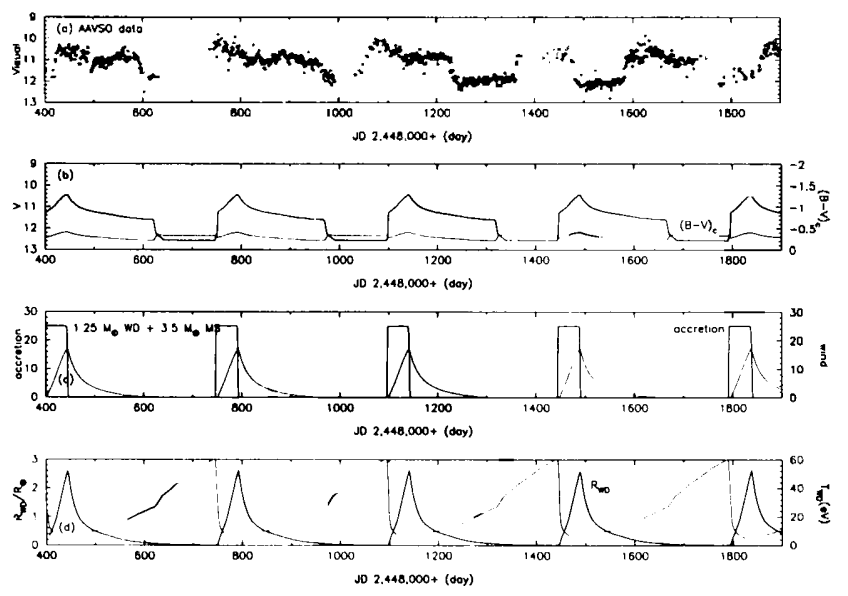

Fig. 4. Long term light curve of the Galactic supersoft X-ray source V Sge for (a) one-day mean of AAVSO observation (taken from Simon \& Mattei 1999), (b) our modeled light curve, (c) mass accretion rate and wind mass loss rate in units of $10^{-6} M_{\odot}, \mathrm{yr}^{-1}$, and (d) photospheric radius and temperature.

lost from the binary. Therefore, the rapid mass accretion is suppressed by the strong wind itself. The mass-stripping rate is roughly proportional to the wind mass-loss rate (Hachisu \& Kato 2003b). As the wind mass-loss rate increases, the mass-stripping rate eventually overcomes the mass-transfer rate. Then, mass accretion to the WD stops.

The mass of the WD envelope is now gradually decreasing, mainly as a result of wind mass loss. The photosphere of the WD is also gradually shrinking. This causes a gradual decay of the optical light curve even if the disk shape is the same. The wind eventually stops. We expect copious supersoft X-rays after the wind stops. The disk shrinks to a normal size from the configuration shown in Figure 2a to that shown in Figure 2b. This causes a sharp $\sim 1 \mathrm{mag}$ drop in the optical light. A rapid mass transfer from the MS to the disk resumes and then the disk edge flares up, as shown in Figure 2c. This phase corresponds to the optical low and X-ray-on state. Thus, the cycle is repeated.

The numerical results are shown in Figure 3. See Hachisu \& Kato (2003b) for more details.

\section{MODELING THE GALACTIC SUPERSOFT SOURCE V SAGITTAE}

$V$ Sagittae also shows quasi-periodic optical high (soft X-ray-off) and low (soft X-ray-on) states with the total period of $\sim 300$ days, longer than RX J0513. A binary model similar to RX J0513 is proposed to explain the long term variation of the light curve. The main differences from RX J0513 are that (1) the mass transfer rate from the MS companion in V Sge is much larger than that in RX J0513 as shown in Figure 4 and that (2) the metallicity in V Sge is about three times larger than that in RX J0513 and the wind is stronger. These make the differences between two objects. See Hachisu \& Kato (2003c) for more details.

\section{REFERENCES}

Alcock, C. et al. 1996, MNRAS, 280, L49

Cowley, A.P., et al., 2002, AJ, 124, 2233

Hachisu, I., \& Kato, M. 2001, ApJ, 558, 323

Hachisu, I., \& Kato, M. 2003a, ApJ, 588, 1003

Hachisu, I., \& Kato, M. 2003b, ApJ, 590, 445

Hachisu, I., \& Kato, M. 2003c, ApJ, 598, 527

Hachisu, I., Kato, M., \& Nomoto, K. 1996, ApJ, 470, L97

Hachisu, I., Kato, M., \& Nomoto, K. 1999a, ApJ, 522, 487

Hachisu, I., Kato, M., Nomoto, K., \& Umeda, H. 1999b, ApJ, 519, 314

Kato, M., \& Hachisu, I., 1994, ApJ, 437, 802

Li, X.-D., \& van den Heuvel, E. P. J. 1997, A\&A, 322, L9

Pakull, M. W., Moch, C., Bianchi, L., Thomas, H.-C., Guibert, J., Beaulieu, J. P., Grison, P., \& Schaeidt, S. 1993, A\&A, 278, L39

Reinsch, K., van Teeseling, A., Beuermann, K., \& Abbott, T. M. C. 1996, A\&A, 309, L11

Reinsch, K., van Teeseling, A., King, A. R., \& Beuermann, K. 2000, A\&A, 354, L37

Schaeidt, S., Hasinger, G., \& Truemper, J. 1993, A\&A, 270, L9

Schandl, S.. Meyer-Hofmeister, E., \& Meyer, F. 1997, A\&A, 318, 73

Simon, V., \& IIattei. J. A. 1999, A\&AS, 139, 75

Southwell, K. A., Livio, M., Charles, P. A., O'Donoghue, D., \& Sutherland, W. J. 1996, ApJ, 470, 1065 